\title{
Design and Application of Real-time Database Model for Smart
}

\section{Residential Quarter}

\author{
Chenlu Tian ${ }^{1, a}$, Shaolin Wang ${ }^{1, b}$,Guiqing Zhang ${ }^{1,2, c}$, and Jiajun Luo ${ }^{1}$ \\ ${ }^{1}$ College of Information\&Electrical Engineering ,Shandong Jianzhu University,Jinan,China \\ ${ }^{2}$ Shandong Provincial Key Laboratory of Intelligent Buildings Technology,Jinan,China \\ a15634135867@163.com, b281419316@qq.com, 'oqzhang@sdjzu.edu.cn
}

Key words: smart residential quarter, distribute, real-time database,SOA,cloud

Abstract:With the development of smart city and smart residential quarter, there are new data demand of time constraint, heterogeneous and distributed data integration, resource sharing ,global management and coordination and regional autonomy. To meet the demands, this thesis reviews the current situation of research on the real-time database for smart residential quarter and puts forward a distributed database model based on SOA. The model is illustrated in database architecture and data model. What's more, A real-time database is constructed based on the model and has been applied in a smart residential quarter. The real-time database provides safe, reliable and efficient data acquisition and processing capability .

\section{Introduction}

Motivated by sustainable development requirements of global environment and modern cities, the concept of the Smart City has been introduced as a strategic device of future urbanization on a global scale[1]. Communities is an essential component of urban services and resident management, and its degree of smartness is closely related to smartness of a city which will finally be reflected in living standard of residents, such as convenience, safety and harmony of life[2]. Smart residential quarter may include or be included in one or more communities. There are many distributed intelligent systems or equipment such as smart homes, environmental monitoring systems, ground-source heat pump system and so on. The intelligent systems or equipments are the data sources in smart residential quarter and create quarter big data as vital part of city big data.

The numerous sensing devices of IOTS and other smart systems generate massive data every second, which is used in the accurate management, real-time control, scientific decision-making of energy-saving operation, regulation of environment comfort degree. There is high real-time requirement in data collection for timely monitoring and control of the environment or electrical facilities. Usually, the database systems such as SQL SERVER or My SQL would not be applicable for the time-critical applications because of the poor real-time performance. Both timing constraints and data consistency would be taken into consider in smart quarter real-time database systems when scheduling the transactions so that they can be accomplished by their corresponding deadlines [3]. Apart from the time constraints that are out of the demand of continuously data tracking, timing correctness requirements are also proposed due to the need to keep data available for the decision-making activities of the controlling systems [4].

At the same time, there are many differences from the industry and electricity power environment. 
1) Smart residential quarter emphasizes regional security and autonomy, there is a great need of global management and coordination associated with regional autonomy.

2) Smart residential quarter apply advanced operating system and support a wide range of platforms and machine language, this make it more convenient and flexible for application construction.

3) Resource sharing: One vital idea of Smart Residential Quarter is resource saving and sharing which include data and service aspects, the data and services acquisition and management are put forward.

4) Multi-level data model: There are various data structures and models which response status of some device or area and adapt to different application needs such as behavior prediction, area state display.

Based on these situations, we put forward a distributed model based on SOA which can meet the demand of time constraint, heterogeneous and distributed data integration, resource sharing, global management and coordination and regional autonomy. This paper will illustrate it in database architecture, data model and classification, service sharing and management.

\section{Related works}

There are several important parts related in the smart residential quarter real-time database including database architecture and model, real time data storage and acquisition, concurrency control, and so on.

Yan $\mathrm{H}$ [5]do a research on database systematic structure, database service and data storage, in the research ,the sensing devices and application write or read the data from database via web-service or other service technology, this means can solve the problem of heterogeneous and platform crossing data transmission ,but web-service technology which is used to collect data will lower the speed of transmission. The distributed databases connected each other through the internet, among which each site has its site autonomy, but it is lack of global management..Stoja S, Jelacic B, Vukmirovic S [6] present the architecture of a real-time database in cloud environment, different distributed services system with in-memory real-time database store different data. The architecture ensures the time constraint and adopts data classification and autonomy idea, but it is lacking in historical data storage and service sharing mechanism.

\section{Design of Real-time database architecture}

The characteristics of the system. In this thesis, we put forward a distributed architecture base on SOA which is adapted to cloud environment or no-cloud environment. The real-time database has the following characteristics:

1) Time-constraint: In the system, we adopt in-memory as the real-time data storage media, memory direct addressing is used to assure the time constraint.

2) Service-oriented architecture: Some service is used to achieve data transmission cross courses and platform, and the service-oriented model is a vital for achieve resource-sharing .

3) Global management and regional autonomy: the data is massive which would be deal with quickly and the data has its ownership, the system has a global management over all of the data and service.

4) Abundant Data models and data classification management: there are abundant data models which meet the various application needs, and adapt the idea of data sorted storing and management . 
Physical Architecture. The system consists of three parts, namely WAN global management server, LAN rational management server, and distributed databases.

Fig. 1 shows the physical architecture of the database system:

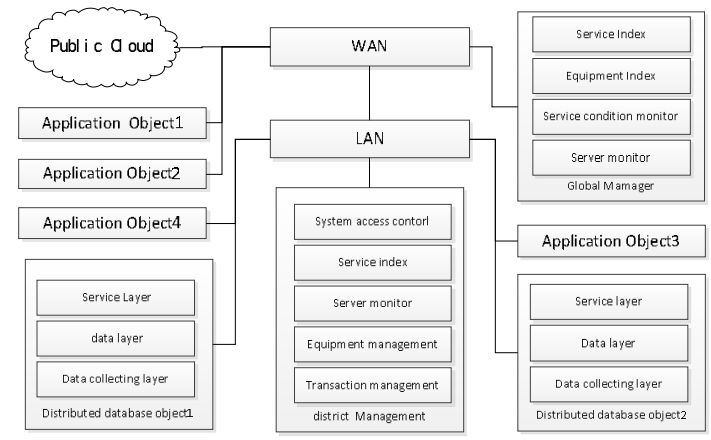

Fig.1 Physical architecture of the database system

\begin{tabular}{|c|c|c|c|c|}
\hline $\begin{array}{l}\text { Rational } \\
\text { manngager }\end{array}$ & $\begin{array}{l}\text { System access } \\
\text { manangement } \\
\text { Service status } \\
\text { recard }\end{array}$ & $\begin{array}{l}\text { Transaction } \\
\text { mmangaments } \\
\text { Service } \\
\text { menitionng }\end{array}$ & $\begin{array}{l}\text { Serviceacecess } \\
\text { manangement } \\
\text { Server tatus } \\
\text { monitioring }\end{array}$ & 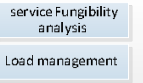 \\
\hline \multicolumn{5}{|c|}{ 令 } \\
\hline \multirow{2}{*}{ Service Layer } & Real time service & Data convertion & Loggirig service & $\begin{array}{c}\text { Service status } \\
\text { monitoring }\end{array}$ \\
\hline & $\begin{array}{l}\text { Historical data } \\
\text { service }\end{array}$ & $\begin{array}{l}\text { Service } \\
\text { moritoring }\end{array}$ & Security senice & $\begin{array}{c}\text { Date } \\
\text { compressing }\end{array}$ \\
\hline $\begin{array}{l}\text { data storage } \\
\text { lyyer }\end{array}$ & 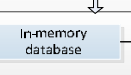 & $\begin{array}{l}- \text { File system } \\
- \text { Sal server/My sql. }\end{array}$ & \multicolumn{2}{|c|}{$\Leftrightarrow \Rightarrow$ Distributed Trensaction manager } \\
\hline \multicolumn{5}{|c|}{ 众 } \\
\hline $\begin{array}{l}\text { ata collecting } \\
\text { layer }\end{array}$ & IOTS Nodes & & diert & API \\
\hline \multicolumn{5}{|c|}{ Distributed database node } \\
\hline Data source & imart hames & GSНP & Securrity System & .... \\
\hline
\end{tabular}

Fig.3 Distributed Database Logical Architecture

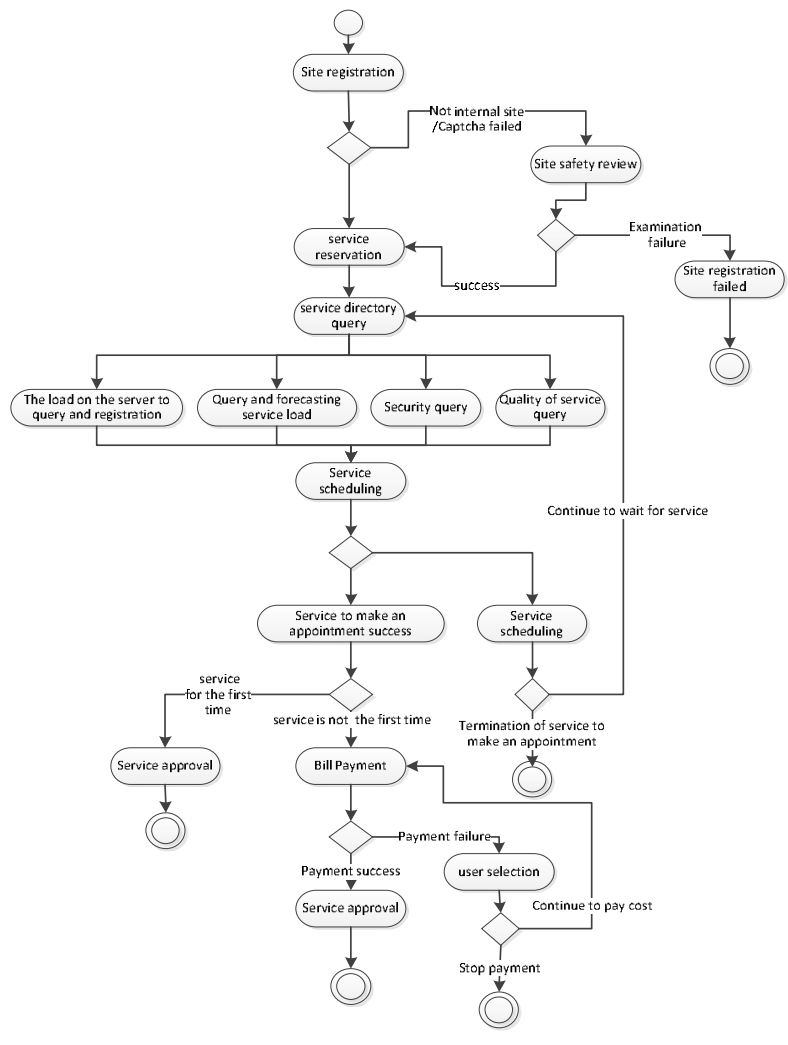

Fig.2 Process of service applying and approval

1) Global management servers: They deploy global service index, all equipment index, service condition monitor and server monitor. The server which keep records of the status of all distributed database objects is the overall guide of the system and is the first access of application of WAN into LAN service. The servers apply online backup technology.

2) Rational management servers: The servers achieves rational service and load coordination, transaction management, they are the real access points of all the application connections and remain connected to the distributed database object and global management servers.

3) Distributed database servers: the objects are distributed to collect the real-time data, store real-time data and some historical data. They provide service interface for data and function.

4) Cloud resources: there are plenty of resources in the cloud such as Iaas, Paas ,Saas, which provide various services. There is a historical data backup mechanism which would upload the distributed object historical data to the cloud file system or other cloud database. What's more ,the system could use the cloud service interface for database application building.

Distributed Database Logical Architecture. District manager is the administrator of local data and services, and manage the authority of distributed database nodes. When some application query service index of global manager, and would like to get a service which located on distributed database node, it must firstly get the unified authorization to LAN, and the district manager controls the access to services or data via the process of site registration, safety review, service reservation, service approval on the basis of permission information, the process is as Fig.2.

Fig.3 shows that every distributed database node consists of four parts, including data collecting layer, data storage layer, service layer and distributed transaction manager. The nodes constitute local cloud. 
1) Data collecting layer: Each data interface is deployed in different areas, as an independent individual which collects real time data, and organizes the data in accordance with the unified communication protocols. The data will be serialized as 2 hexadecimal data streaming which will be transmitted to the data storage layer.

2) Data storage layer: There are in-memory and hard disk storage medium. In-memory database store real-time data and some historical data of recent period of time. historical data is stored in the file system or some RDBMS. The related services in service layer are called for the data processing such as data analysis and storage.

3) Service layer: Service layer includes a variety of services which collaborate with each other and achieve the main functions of the real-time database, provide some ancillary services such as data conversion, data mining. The services in service layer can be divided into two classes according to different data sources and has different boarding and realization mode. If the source of service is local in-memory database or local disk data, the service can only be located on the local server to provide functions of extracting, transforming of local data, another type of services whose data source can be external data without consideration of data location. The model can well solve the load balancing problem under distributed environment of smart residential quarter, when rational manager monitors the servers' load and find access load of some server is too high, it would allocate some tasks to other database services, as a result, it will reduce the server load. This method would improve the execution efficiency .

4) Distributed transaction manager: The distributed transaction manager accepts the tasks from district manager and arranges them according to the priority .Services cooperate to achieve a transaction, the services deployed locally is transferred preferentially.

\section{Data analysis and model}

data analysis. Data objects should be fully analyzed for reasonable data model establishment. Data analysis includes value type and operations analysis.

1) Value type analysis: The data in the smart residential quarter could be divided into two types.

Dynamic data: This type of data has time constraint, includes direct real time data and derived data .The data reflects status of a device, intelligent system or some area.

Static data: The data includes relationship, user authority and other layout data which would not always change over time.

2) Operation analysis: The measuring point value is the basic management information model of Real-time database .Real-time data of the measuring points is from the IOTS nodes and OPC client, but a IOTS node often manage a room or a group of associated points which depict a certain room or environment status, at the same time, in the upper application, multiple algorithms such as comfort algorithm would directly use part or all value of a room. Therefore, the establishment of the data model should consider completeness, scalability, and the connections between the measuring points, etc.

Data model. The database provides basic storage model, IOTS equipment model, user preference model, configuration model, equipment deployment model. The structures are constructed based on object.

1) Basic storage model: The model is the basic storage of dynamic data, could be described as a triad<time,value,datatype $>$, if there is the need of recording the data quality, the model could be described as <time, value, datatype, quality>. The value is Stored in binary data format, datatype is enumerated values. 
2) Equipment model: The equipment model could be understood as a relevant point sets, such as air condition structure, environment equipment, intelligent switches structure, intelligent socket. The model is object-based, environment structure are listed as $<$ ValueTime, Humidty ,Temperature, LightDense, CO2Concentr, Infrared >.

3) Configuration model: configuration model describe the device or deployment static attributes. There are three configuration models: measuring point model ,equipment model, network model, district deploy model. The models has correlations , Fig.4 shows the relations. The server, device and measuring point have attribute named relation, the relation could be realized via two-dimensional array which store the network connected points(server,measuring point, device) and entered communication data volume.

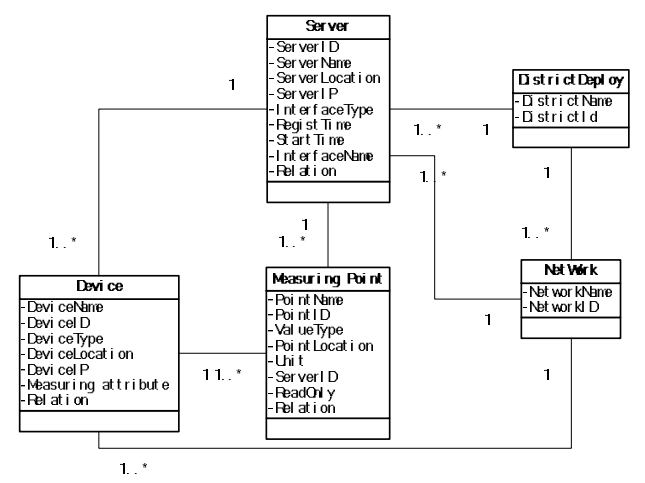

Fig.4 Correlation of configuration models

\section{Typical Application}

The database has been applied in a smart residential quarter which covers an area of 120000 square meters. The residential quarter deploys smart home, GSHP, environment monitoring systems and so on. There are about 40000 points in the residential quarters.

The database is developed based on .net platform which provides many advanced technologies for SOA realization such as .net remoting, WCF, multi-threading. The services development mainly uses WCF that provides Security mechanisms including transfer security, authorization or access Control, auditing.The database store integrated data, some independent devices or sensors data.

We divide the dynamic data into three classes according to the data type and data acquisition type,they are OPC data,IOTS data,indepent data.And we set up different storage area to store different data classes.

For IOTS node,the data is stored in the in-memory by means of measured points set,the set are organized in accordance with specific rules.Taking into and the data parser, data transfered every time is the same equipment's data , and the data provided to the outside application is firstly tansformed to the specific data struct, then be serialized to binary streams .For OPC data,the data is stored in the in-memory by means of independent measured point,data transfered everytime is the same data type. And for the independent devices, it is the same as the opc data,the data is stored by means of indepent measurd point,and we set up visual serverID for this class data,the storage struct is as Fig. 5 shows.

What's more, SQL SERVER is chosen as history database, and we developed some web-services for some specific data acquisition need. 


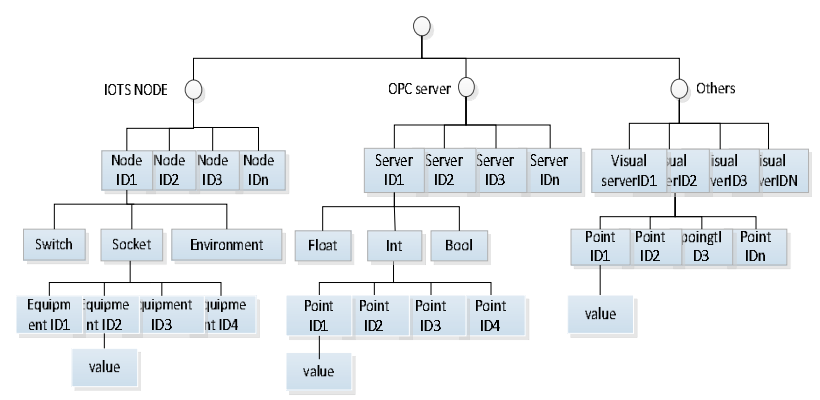

Fig. 5 In-Memory Storage Structure

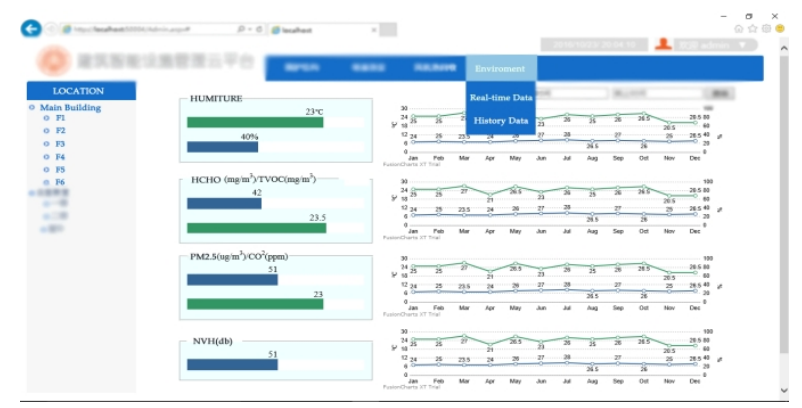

Fig. 6 Environment remote monitoring web page

The Fig. 6 shows a web page of equipment information and management system which is an important part of smart residensial information platform, the page realizes its remote environment monitoring of different locations based on our database. The application declares our database model's availability and the real-time database provides safe, reliable and efficient data acquisition and processing capability .

\section{Conclusion}

This thesis analyzes the demand smart residential quarter and puts forward a distributed database model based on SOA. A real-time database is constructed based on the model and applied in a smart residential quarter. The real-time database provides safe, reliable and efficient data acquisition and processing capability .

\section{Acknowledgements}

This work was financially supported by the Natural Science Foundation of China(61573225), Scientific Research and Development Projects of Shandong Wall Materials Innovation and Building Energy Conservation(QG021)

\section{Reference}

[1]Wang J, Chao L, Zhang X, et al. Survey of Data-Centric Smart City[J]. Journal of Computer Research \& Development, 2014, 51(2):239-259.

[2]Yan H, Long D. Research on Key Technology for Data Storage in Smart Community Based on Big Data[C]// International Conference on Intelligent Transportation, Big Data and Smart City. IEEE, 2015:653-656.

[3] Son S H, Poris M S, Iannacone C C. Implementing a Distributed Real-Time Database Manager[C]. DASFAA. 1991: 51-60

[4] Ramamritham K, Son S H, Dipippo L C. Real-time databases and data services[J]. Real-Time Systems, 2004, 28(2-3): 179-215

[5] Yan H, Long D. Research on Key Technology for Data Storage in Smart Community Based on Big Data[C]// International Conference on Intelligent Transportation, Big Data and Smart City. IEEE, 2015:653-656.

[6] Stoja S, Jelacic B, Vukmirovic S, et al. Architecture of Real-Time Database in Cloud Environment for Distributed Systems[C]// International Conference on Artificial Intelligence, Modelling and Simulation. IEEE, 2014. 